\title{
Comparative study on Image Segmentation and Classification Analysis for brain Abnormality
}

\author{
Shreyash Subhash Sharma, M.E Scholar, Thakur College of Engineering and Technology, \\ Mumbai, India, shreyashssharma@gmail.com \\ Dr. Megharani Patil, Associate Professor, Thakur College of Engineering and Technology,
} Mumbai, India, megharanitpatil@gmail.com

\begin{abstract}
Brain abnormal is one of the most dangerous disease occurring commonly among human beings. There are many diseases such as Alzheimer's Disease, Dementias, Epilepsy and other Seizure Disorders, Mental Disorders, etc. due to small abnormalities captured in MRI. The MRI brain abnormality segmentation is an important technique in medical diagnosis. Due to large variance and complexity of abnormal characteristics such as size, location, intensity and shape in MRI images, prediction of abnormal region is very complex. So currently manual tracing and delineating of segmentation of brain abnormality is in practice. The study of image segmentation and classification is done to improve the quality of image to train and classify different morphological functions. Accuracy is measured for the classification process and the classifier model can be found by comparing the accuracies obtained. It will be described the network building algorithm, chosen practical field for proposed method application and showed the results of its programming implementation.
\end{abstract}

Keywords - CT, MEG, EEG, PET, MRI, K-means, FCM, SVM, ANN, SPECT, SBRG, TIA.

\section{INTRODUCTION}

Raymond Vahan Damadian (born March 16, 1936) is an American physician, medical practitioner, and inventor of the first MR (Magnetic Resonance) Scanning Machine. Image segmentation is one of the primary steps in image analysis for identifying the brain tissues. The brain abnormal detected images process classified into different image classification since treatment planning varies for different types of abnormalities ${ }^{[1]}$. The intervention of human perception performed the detected process manually which is highly prone to error. The image segmentation algorithms play an active and dominant role in image analysis, image retrieval, image understanding and image processing. It also plays a vital role in a lot of biomedical imaging applications ${ }^{[2]}$. This brain abnormality can be an essential tumor or a metastasis from a abnormal in another organ. Abnormality segmentation from MRI data is an important but time-consuming manual task performed by medical experts. The MRI T1-weighted and T2- weighted images are the most commonly used techniques MRI imaging. The MRI brain tumor segmentation provides useful information for surgical planning and further medical diagnosis ${ }^{[3]}$. The large variance and complexity of tumor characteristics such as size, location, intensity and shape in MRI images is a difficult task. So the manual tracing and delineating of segmentation of brain tumor is in practice. The segmentation is a significant tool in medical image dispensation and it has many applications, in discovery of abnormal, measuring tumor volume, surgical planning and preparation, discovering coronary edges, categorization of blood cell. Imaging modalities like X-ray, CT (ComputedTechnology), MRI(Magnetic-Resonance-Imaging) etc. have important role to play towards evaluation of patients having abnormal functioning of brain. It improves ability of clinical doctors to study the mechanism of brain abnormality for better treatment ${ }^{[4]}$. Brain tumor segmentation is to segment abnormal tissues such as active cells, necrotic core, and edema from normal brain tissues.

\section{LITERATURE REVIEW}

The objective of this review section is to present literature survey of image segmentation methods. Hapsari Peni Agustin Tjahyaningtijas,2018 ${ }^{[5]}$ purposed classification methods of brain tumor segmentation. As per him, segmentation shall be classified into three categories i.e. manual, semiautomatic, and fully automatic, depending on the quantum of human interaction required. The semiautomatic and fully automatic segmentation of tumor brain images need improvements as they show partial volume effects of brain tumor. To make acceptable automated Brain Tumor segmentation, Positron Emission Tomography (PET), Magnetic Resonance Spectroscopy (MRS) and Diffusion Tensor Imaging (DTI) may help. 
Amrutha Ravi, Sreejith S., $2015^{[6]}$ purposed that an automatic brain tumor detection system selecting a suitable segmentation method. But there are several difficulties that cause poor segmentation results. The main difficulties in segmenting an image are Noise, Blur Low Contrast, the bias field, the partial volume effect. Therefore, improvements needed in current methods are correcting the data for unwanted atmospheric noise, removal of non-brain element image and converting the data so they correctly reflected in the original image. The identification of a good threshold on which this method relies, if fails, may guide to poor segmentation.

Jin Liu, Min Li, Jianxin Wang, Fang xiang Wu, Tianming Liu, and Yi Pan, $2014^{[7]}$ proposed that conventional brain tumor segmentation methods mainly include the use of standard image processing methods such as threshold-based methods and region-based methods. The Jaccard coefficient and the Dice Similarity Coefficient (DSC) can range from 0 to 1 with 0 indicating no overlap and 1 indicating perfect overlap. They are of the view that Robustness of Segmentation method, Computation time and real-time segmentation needs improvement.

\section{PROPOSED SYSTEM}

The purposed work is used to observe the methods utilized are filtering, contrast adjustment, negation of an image, image subtraction, erosion, dilation, threshold, and outlining of the brain abnormal. To provide information like size, dimension and position of the tumor, plotting contour of the abnormal and its boundary provides with information related to the tumor that can prove useful for various cases which will provide a better base for the staff to decide the curing procedure. To bring some useful information in simpler form in front of the users, especially for the medical staff treating the patient. Magnetic resonance images are observed and detected the size and location of brain abnormal. To demonstrate knowledge including signs and symptoms, methods of detection and patterns of spread using the image segmentation.

\begin{tabular}{|c|c|}
\hline Feature Extraction Application & $\begin{array}{c}\text { MATLAB r2015a, SciLab, NumPy, } \\
\text { Sklearn and other deep learning } \\
\text { tools. }\end{array}$ \\
\hline Classifiers & MATLAB r2015a \\
\hline Operating System & Windows 10, 64-bit \\
\hline Processors & $\begin{array}{c}\text { Intel } \text { Core }^{\mathrm{TM}} \text { i5-7300HQ } \\
\text { CPU@ } 2.4 \mathrm{GHz}\end{array}$ \\
\hline RAM & $8 \mathrm{~GB}$ \\
\hline
\end{tabular}

Table 1: Technology Required

In table 1, this paper is implementing in MATLAB image processing for analyzing and classifying the image segmentation.

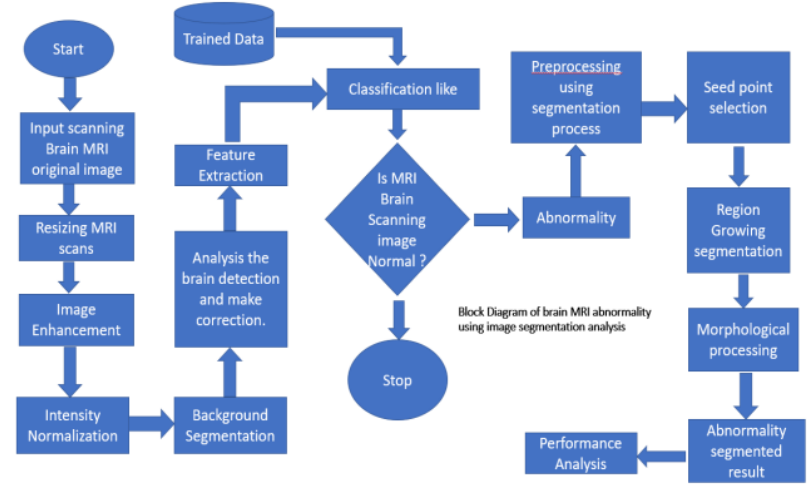

Figure 1: Block Diagram

Input scanning Brain MRI original image is collecting the brain MRI image from patient and read it. Resizing MRI scans are resizing the volume intensity of brain MRI image. It returns the volume that has the number of rows, columns, and planes specified by the three-element vector. Intensity Normalization suggests a method where a set of standard histogram landmarks are learned from a set of MRI images. These landmarks are then used to equalize the histograms of the images to normalize ${ }^{[8]}$. Background Segmentation method depends upon the selection of a certain value of the threshold, which needs to be good otherwise, this can lead to a trivial or inconsequential segmentation. To add further, thresholding is known to be the commonly used first step in a series of operations involving image processing. Background Segmentation corrects the images. Feature extraction helps to train the dataset in analyzing the regression and validation on comparison of various algorithms on MATLAB in the following table $1^{[9]}$. Abnormality finds small abnormality inside the brain images after classifying the feature extraction. In SBRG (Seed-based region growing) process, a segmented image is generated from a point called seed. The region, beginning with this seed, grows by appending to another nearby seed whose adjacent pixels have properties which are similar to the seed. Morphological operators create a binary image and a structuring element as input then its combine using a set operator. Input image based on characteristics of its shape which are encoded in the structuring element process objects by them. These operators also are combining of erosion and dilation to select features of a certain shape. Abnormality segmented result analysis and detects the brain abnormal images to give the result using image segmentation. Performance Analysis performs the brain abnormal image to find out the result of region growing, edge detection and morphological processing like dilation and erosion.

\section{IMPLEMENTATION}

It can input to create the original image to display the output below figure 2 . Its analysis the image to design the image segmentation and classification to build the programs. 


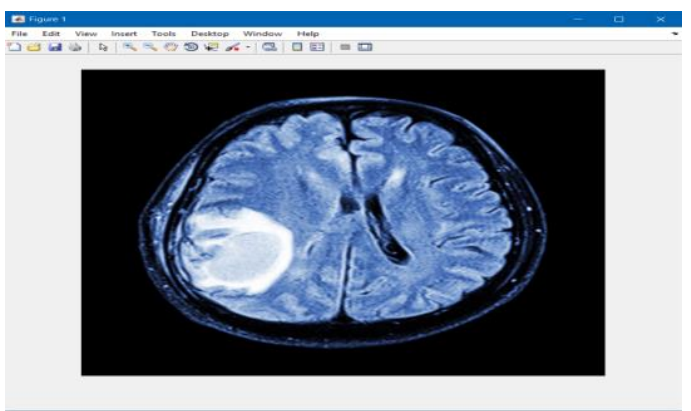

Figure 2: Original image

It converts into grayscale image in the output. Grayscale is a range of shades of gray without apparent color. The darkest possible shade is black, which is the total absence of transmitted or reflected light below figure 3 .

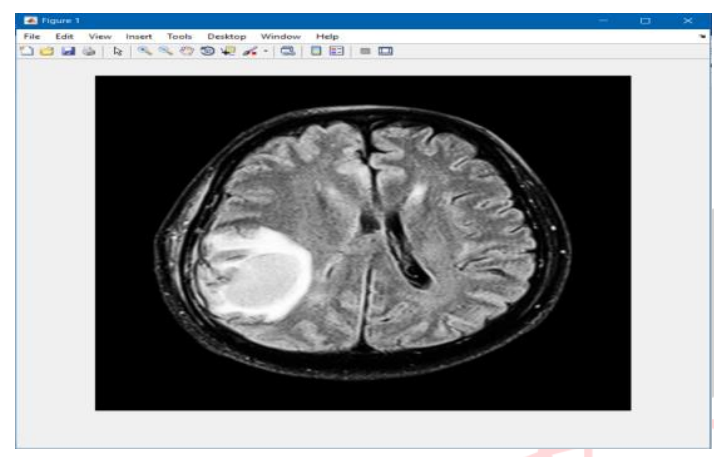

Figure 3: Grayscale image

\section{A. Thresholding segmentation}

Thresholding algorithm is very simple to display image processing after analyzing the greyscale image. These algorithms computes to display both background and foreground with the help of pixel information. It gives smooth image but not the histogram using the noisy images ${ }^{[10]}$. It can be divided into 2 categories - global thresholding and adaptive thresholding image. The global thresholding choose threshold that separates object from background. The global threshold selection has been aided with the use of local properties of image below figure 4 .

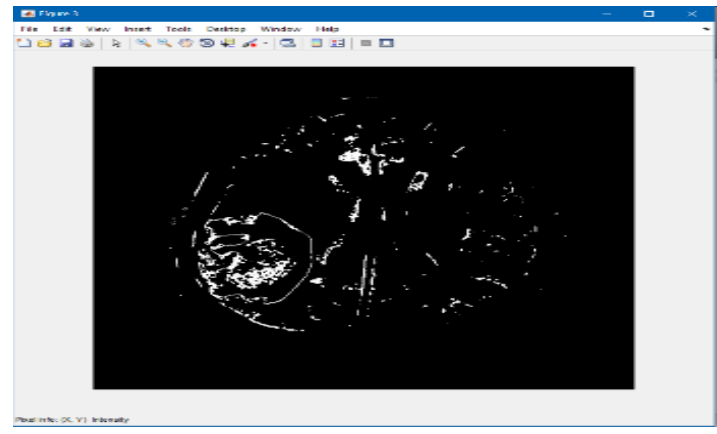

Figure 4: Global thresholding image

Simple thresholding is not possible for many objects at different greyscale level and variations in background greyscale level. Adaptive thresholding is used to display thresholding images and divide image into regions. It performs thresholding independently in each region.
Different threshold values for different local areas are used in Adaptive thresholding below figure 5.

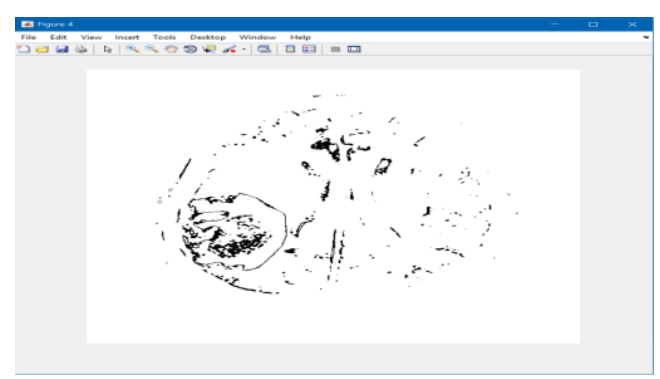

Figure 5: Adaptive thresholding image

The drawback of this method is that it is computational expensive and, therefore, is not appropriate for real-time applications.

\section{B. Removal Noise using Dilation and Erosion}

It displays to binarize 0.8 less than 1 value of threshold image to reduce noise on brain abnormal where it is converting from grayscale image into thresholding image. It removes the noise using morphology functions. It removes pixels which do not belong to the objects of interest using background. A dilation operation is performed on a binary image to fill in the holes in the image. See below figure 6 .

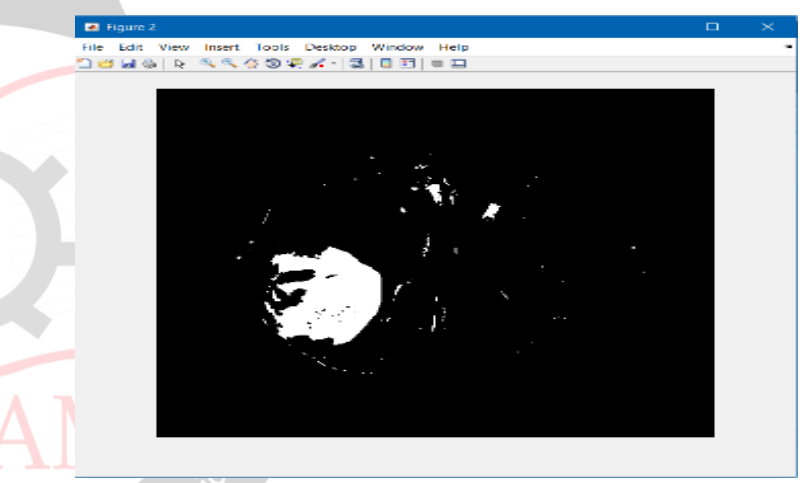

Figure 6: Remove noise using Dilation

Operation of Erosion is opposite to dilation. Erosion reduces boundaries and increases size of holes. See below figure 7 .

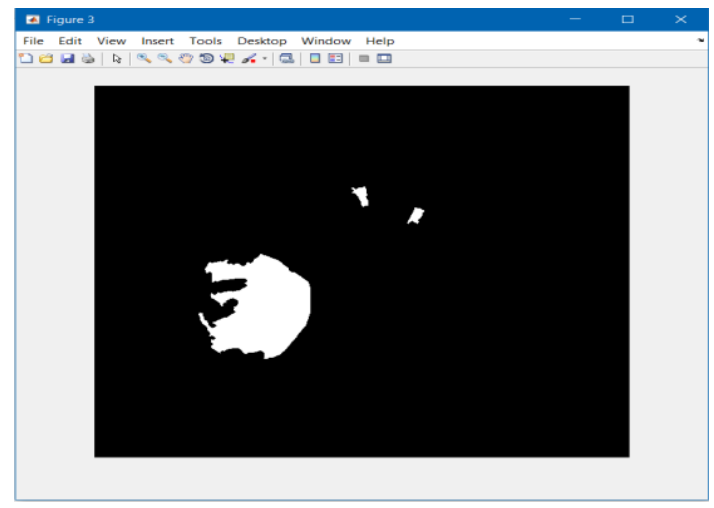

Figure 7: Removing noise using Erosion

It shows an example of how erosion shrinks boundaries of an image and enlarges holes. Erosion can also be used to reduce noises from an image. 


\section{Region growing based image segmentation}

Segmentation of images is crucial to our understanding of them. These techniques have been proposed and tried for segmenting images by identifying regions of some common property. The techniques of merging and splitting algorithms are used for segmentation of images. In the technique of merging algorithms, comparing of adjacent regions is done and if they are close enough in some property, they will be merged ${ }^{[11]}$. In the technique of splitting algorithms, huge big regions, specially that are uniform, are split into smaller uniform or non-uniform areas, but preferably into uniform smaller areas below figure 8 .

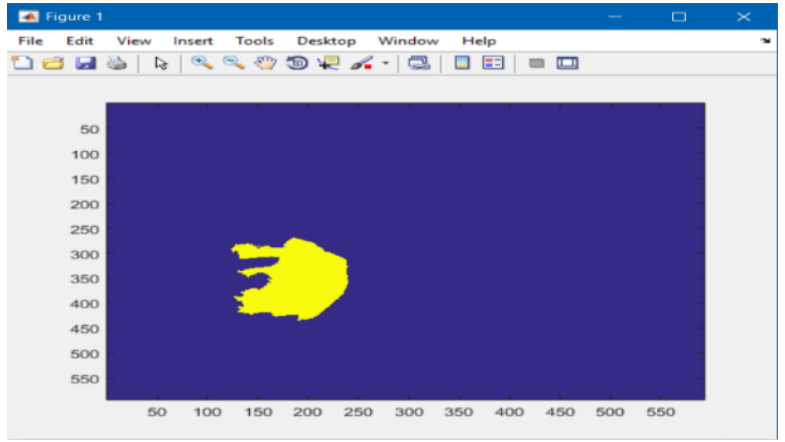

Figure 8: Region growing based on image segmentation

Uniformity criteria can be set by using variance or standard deviation or other statistical techniques. It displays result of active contour model based on region growing of image segmentation. This thresholding process produces rigid boundaries and many small particles, does not belong to the object below figure 9 .

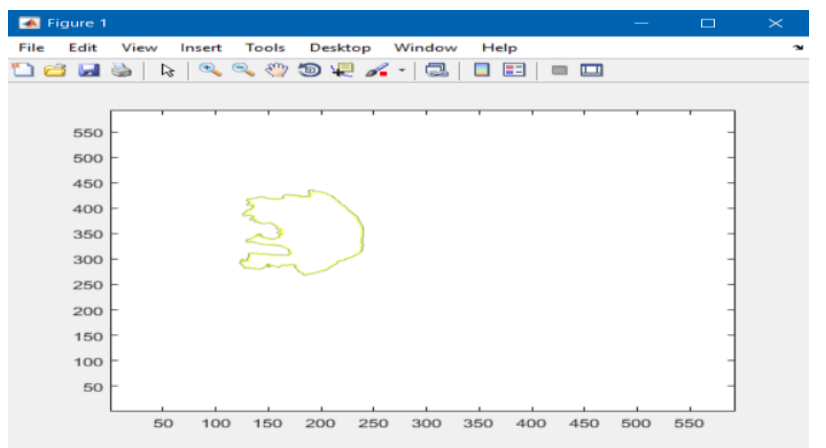

Figure 9: Active contour of region growing

\section{Watershed segmentation}

Watershed segmentation is used to transform gray scale image which is an important region-based segmentation of brain abnormal how to display the image segmentation for identifying the selection seed of brain abnormal. The gradient magnitude improves grayscale image. See below figure 10. The gradient is high at the borders of the objects and low inside the objects.

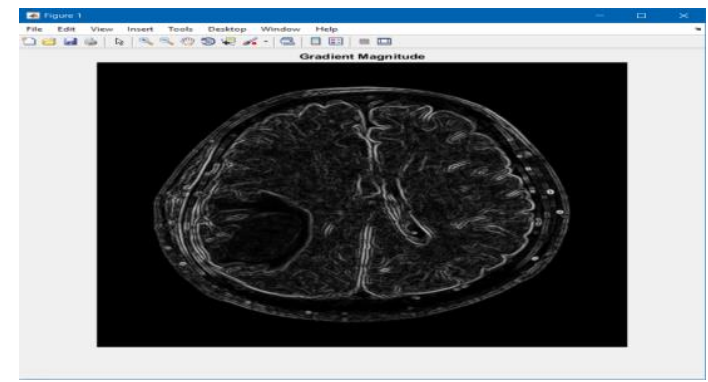

Figure 10: Gradient Magnitude

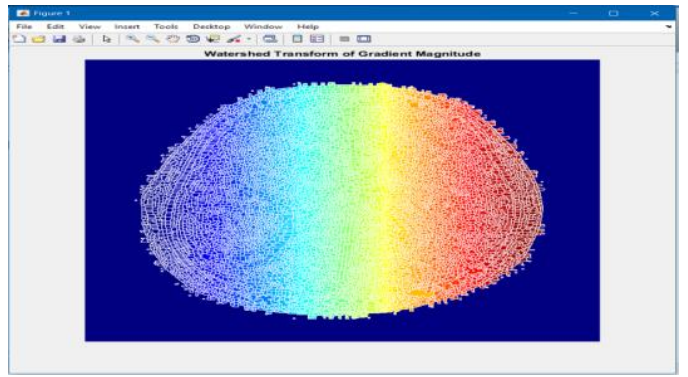

Figure 11: Watershed transform

Watershed transformation is done to display color segmentation from grayscale where the line is marked in outline of brain abnormal. See above figure 11. The type of morphological techniques such as opening and closing by reconstruction is used to clean the images in figure 12,13 , $14,15$.

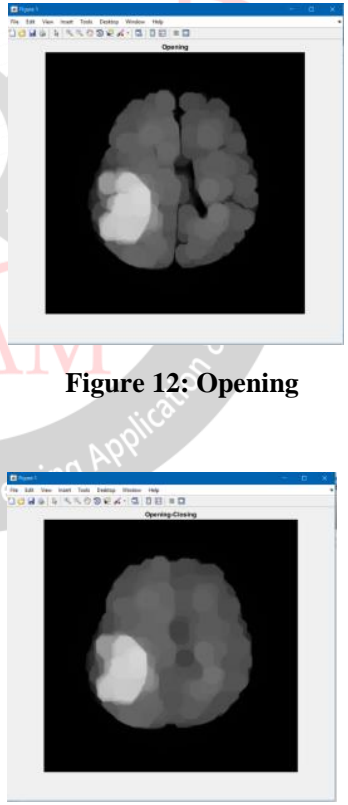

Figure 14: Opening-closing

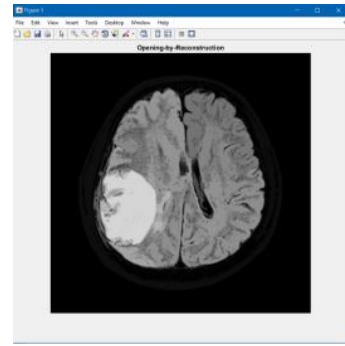

Figure 13: Opening by

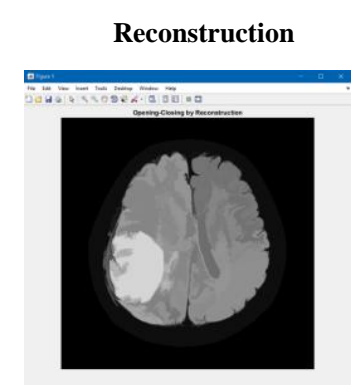

Figure 15: opening-closing

By reconstruction
It compares different reconstruction-based opening and closing to display the watershed segmentation and are more effective than standard opening and closing at removing small mark without impact on complete shape of the brain abnormal images ${ }^{[12]}$. It also calculates to display the regional maxima output in figure 16. 


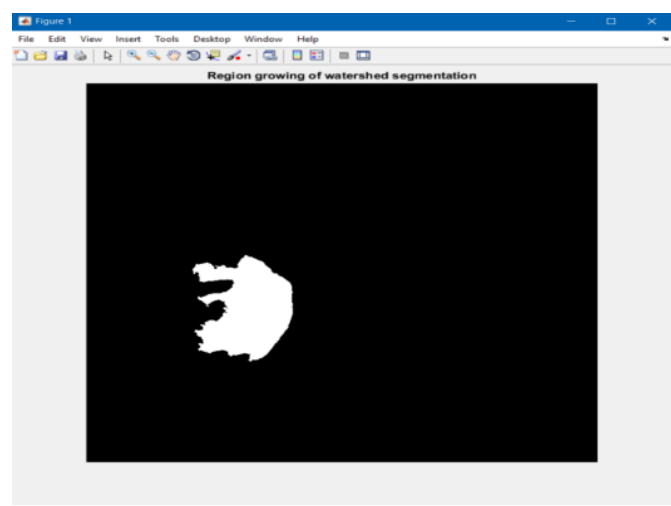

Figure 16: Region growing of watershed segmentation

\section{E. Brain abnormal detection using K-means and FCM algorithms}

It helps to detect brain abnormal image to calculate metrics, perimeter, boundary and centroids using K-means and FCM (Fuzzy C- Means) classifications using methodological detection. It also calculates the weight of detected brain abnormal to measure brain infected tissue like small part of region growing $0.28,0.46$ and 0.51 weight marked to analyzing the detected brain abnormality in figure 17. Detecting brain tumor cell can potentially save millions of lives ${ }^{[13]}$. The shape of the tumor cell plays a vital role in determining the severity of tumor which can be identified using image classification algorithms.

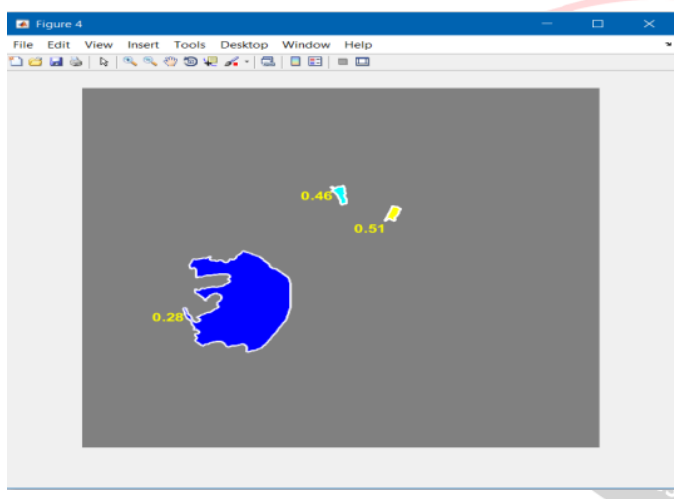

Figure 17: Kmeans algorithm detected result

FCM algorithms-based method of detection is used to display image segmentation and correct the objective functions and centers to improve the quality of image as shown in figure 18 .

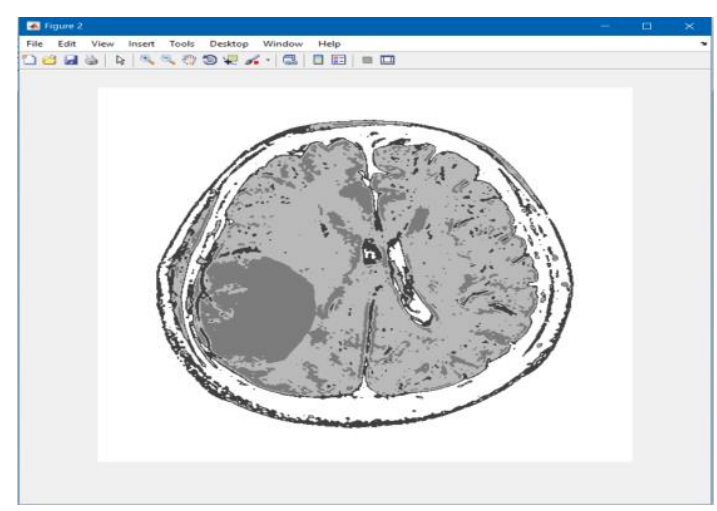

Figure 18: FCM algorithm detected result

\section{RESULT}

This algorithm performs to calculate and train the image segmentation on time-consumption, speed performance while correcting the image segmentation using the program implementation on MATLAB.

\section{A. K-means algorithm result}

It finds to calculate centroid, area, perimeter, metrics and boundary to analysis the brain abnormal detection result. The centroid is a point obtained as a weighted average of pixel coordinates, where weights are the intensities. It also calculates area of detection result as shown in table 2.

$\begin{array}{r}\hline \text { Area } \text { 占 } \quad \text { Centroid } \\ 13263[184.1283,353.1773] \\ \hline 321[296.2336,175.1807] \\ \hline 259[356.5251,207.1969] \\ \hline\end{array}$

Table 2: Area and centroid

To measure the quality of the image is Signal-to-Noise Ratio (SNR) which is the ratio of noise-free image contrast to the noise contrast. Image quality can degrade due to distortions during image acquisition and processing. It also calculates threshold value, boundary of $\mathrm{x}$ and $\mathrm{y}$ using image segmentation while running the brain abnormal detection result in figure 17 . Metric $=4 * \mathrm{pi}^{*}$ area/ $/$ perimeter ${ }^{\wedge} 2$.

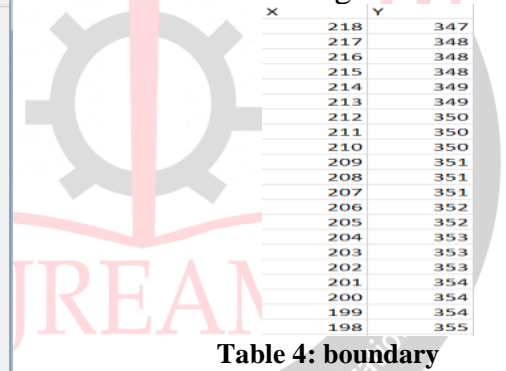

\section{Perimeter 79.84062}

Table 5: perimeter Table 6: Threshold It Computes perimeter value as 79.8406 , threshold value as 0.9400 while calculating the boundaries. See above table 4 , 5,6 .

\section{B. FCM algorithm result}

FCM algorithm helps to test the objective functions and calculates to measure the centers in the area of brain abnormal image using the image segmentation analysis in table 7,8 .

\begin{tabular}{|rrr|}
\hline x & \multicolumn{1}{l}{$k$} & options \\
0.45546 & 0.546363 & 0.747982 \\
0.183409 & 0.232483 & 0.334461 \\
0.850176 & 0.86991 & 0.942766 \\
0.007698 & 0.011065 & 0.028779 \\
\hline
\end{tabular}

It computes to calculate the iteration count and objective function values to measure the brain abnormality result with the help of FCM algorithm as shown below table 9. 


\begin{tabular}{|c|c|}
\hline Iteration count $=1$, obj. fcn $=32862.635051$ & Iteration count $=16$, obj. fcn $=2407.760743$ \\
\hline Iteration count $=2$, obj. fen $=25052.420634$ & Iteration count $=17$, obj. fcn $=2400.505383$ \\
\hline Iteration count $=3$, obj. $\mathrm{fcn}=25045.988252$ & Iteration count $=18$, obj. $\mathrm{fcn}=2395.330535$ \\
\hline Iteration count $=4$, obj. fcn $=24950.668013$ & Iteration count $=19$, obj. $\mathrm{fcn}=2391.585177$ \\
\hline Iteration count $=5$, obj. fcn $=23642.327221$ & Iteration count $=20$, obj. $\mathrm{fcn}=2388.836922$ \\
\hline Iteration count $=6$, obj. fcn $=13846.945658$ & Iteration count $=21$ obj. $\mathrm{fcn}=2386.794085$ \\
\hline Iteration count $=7$, obj. $\mathrm{fcn}=4776.432743$ & Iteration count $=22$, obj. $\mathrm{fcn}=2385.257166$ \\
\hline Iteration count $=8$, obj. $\mathrm{fcn}=3531.011854$ & Iteration count $=23$, obj. fcn $=2384.087916$ \\
\hline Iteration count $=9$, obj. $\mathrm{fcn}=2906.651053$ & Iteration count $=24$, obj. fcn $=2383.189276$ \\
\hline Iteration count $=10$, obj. $\mathrm{fcn}=2651.737608$ & Iteration count $=25$, obj. $\mathrm{fcn}=2382.492223$ \\
\hline \multicolumn{2}{|l|}{ Iteration count $=11, \mathrm{obj} . \mathrm{fcn}=2545.462363$} \\
\hline \multicolumn{2}{|l|}{ Iteration count $=12$, obj. $\mathrm{fcn}=2489.484214$} \\
\hline Iteration count $=14$, obj. $\mathrm{fcn}=2433.087941$ & \\
\hline rat & \\
\hline
\end{tabular}

Its analysis the implementation to show all overall experimented result in the program to improve the quality of the output image.

\section{Performance Comparison of K-means and FCM algorithms}

It performs image segmentation with the FCM (Fuzzy C Means) algorithm results of which can be seen in above figure 18. Computation of the centers to functions of $\mathrm{x}, \mathrm{k}$ and options are performed in Table 7. It helps to train the neural network on MATLAB while checking the validation result with the help of regression analysis in figure 19. Performance of regression analysis is $97.76 \%$.

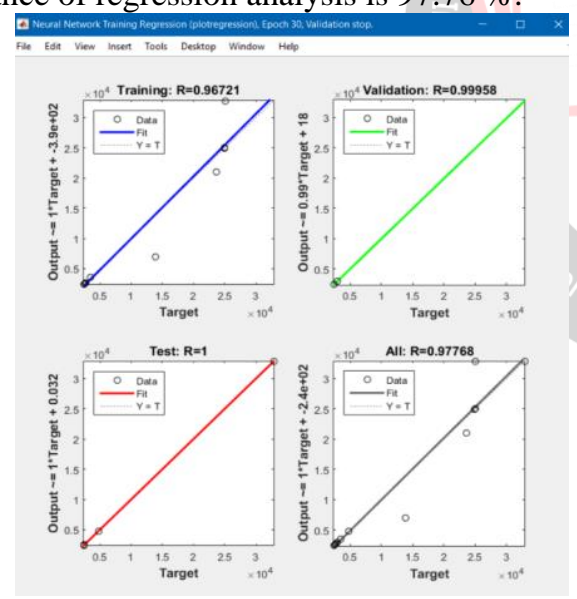

Figure 19: Validation of FCM

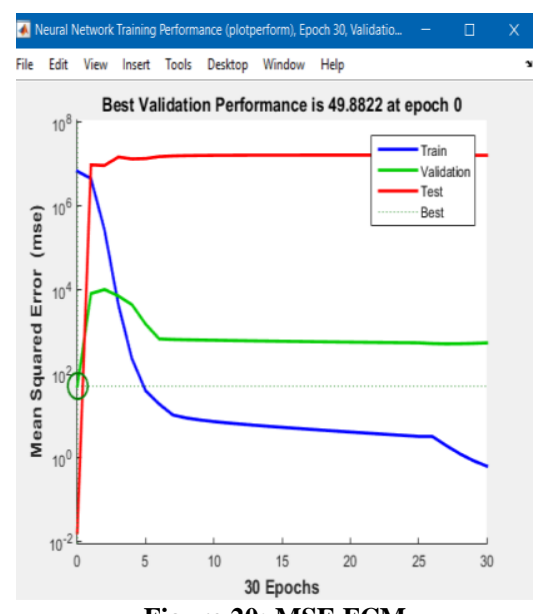

Figure 20: MSE FCM

It also performs image segmentation with the help of neural networks on MATLAB using k-means algorithm. It also compute the centroids to divide $\mathrm{C} 1, \mathrm{C} 2, \mathrm{C} 3$ and $\mathrm{C} 4$ and area above table 2. Regression analysis helps it to perform validation result $(89.82 \%)$ as shown in below figure 21 .

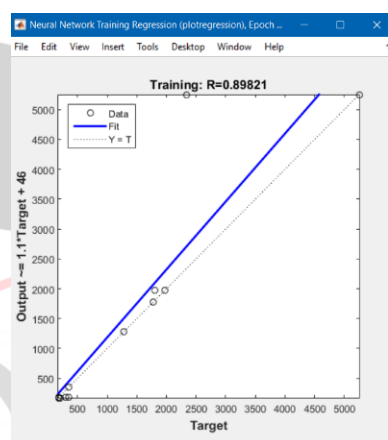

Figure 21: Validation

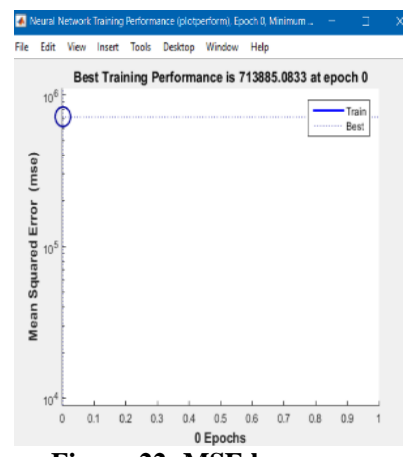

Figure 22: MSE k-means
It trains the best performance of validation result to analysis the MSE and calculate its result $(71.38 \%)$ in k-means algorithm as shown in above figure 22 .

It assumes to put validation checks $30 \%$ and epochs to analysis the Mean Squared Error (MSE) of image segmentation on both the algorithms. It marks the best validation performance of Neural Network (NN) Training dataset in figure 20. 


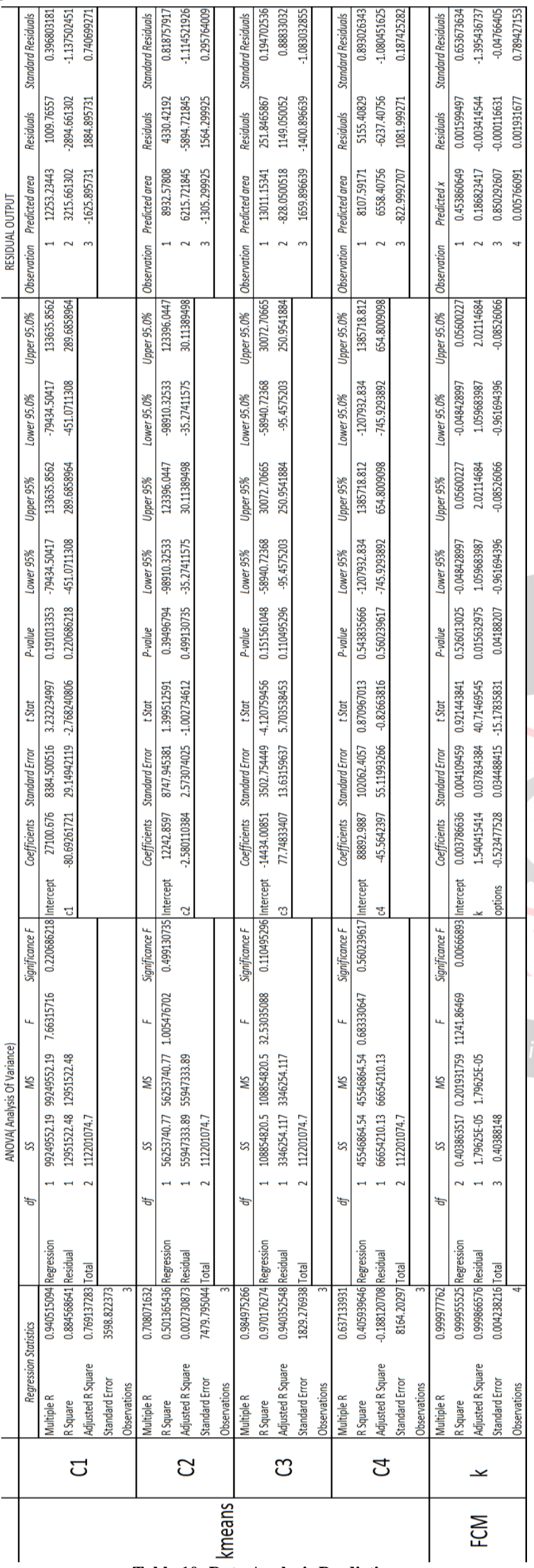

Table 10: Data Analysis Predictive
Data analysis predicted to calculate the regression statistics such as Multiple R, R Square, Adjusted R Squared and standard error using both the algorithms. ANOVA (Analysis of Variance) helps to calculate both algorithms such as df (degree of freedom), SS (Sum-of-Square), MS (Mean Square), F, Significance F. It's also calculated the data analysis of coefficients, t Stat, P-value, lower $95 \%$ and upper $95 \%$. Residual output of both algorithms predicted the calculation of predicted area, residuals and standard residuals in the given table 10. Overall performance of data analysis predictive result is used to calculate k-means algorithm below figure 23 and table 11 .

\begin{tabular}{rr}
\hline Percentage & \multicolumn{1}{c}{ area } \\
\hline 16.66666667 & 259 \\
50 & 321 \\
83.33333333 & 13263 \\
\hline
\end{tabular}

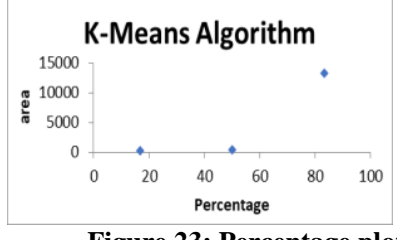

Figure 23: Percentage plot

FCM algorithm is used to calculate overall performance of data analysis predictive result below figure 24 and table 12 .

\begin{tabular}{rc}
\hline \multicolumn{1}{c}{ Percentage } & $x$ \\
\hline 12.5 & 0.007697769 \\
37.5 & 0.183408873 \\
62.5 & 0.455460146 \\
87.5 & 0.850175976 \\
\hline
\end{tabular}

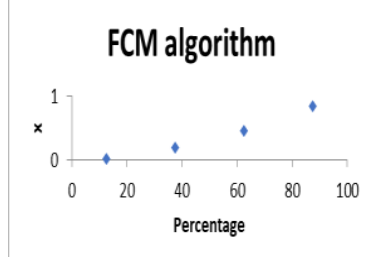

Figure 24: Percentage plot

Both k-means and FCM algorithms showed the percentage of data analysis predictive are $83.33 \%$ and $87.50 \%$. Hence both the neural network dataset training and data analysis result of FCM is better than k-means algorithm but time consumption of $\mathrm{k}$-means is less and results are also smoother than FCM algorithms.

\section{CONCLUSION}

This paper concluded that the comparative study on image classification and segmentation analysis are providing the information to train and detect the validation result for displaying quality of high and low output images. Thresholding, Region and Watershed based image segmentations are discussed. Under the review of the thresholding algorithm, it must remove the noise using dilation and erosion with the help of image segmentation. Comparison of k-means and FCM algorithms are discussed and performed to train the validation result in the neural network dataset training and data analysis predictive. It is improving the signified knowledge of predicting image segment and classify the ground selection seed of brain abnormal by physicians. Results and analysis show that the proposed approach is a valuable diagnosing technique for the physicians to detect the brain tumors. Therefore, in order to improve the accuracy in the segmentation, it is necessary to include additional knowledge for discarding other tissues [10]. The designs that are experimental in 
nature normally aim to collect carefully minimally sufficient amount reasonable data to be able to estimate model parameters by providing enough information.

\section{REFERENCES}

[1] Shaikh Abdul Hannan, Bharatratna P. Gaikwad, Ramesh R. Manza, "Brain tumor from MR Images: A Review", International Journal of Scientific \& Engineering Research, Volume 5, Issue 4, April-2014 1325, ISSN 2229-5518.

[2] Xiao Xuan, Qingmin Liao, "Statistical Structure Analysis in MRI Brain Tumor Segmentation", DOI: 10.1109/ICIG.2007.181, IEEE Xplore, Image and Graphics, 2007. ICIG 2007.

[3] Maha Lakshmi, Velmurugan T, "Segmentation of MRI Brain Images: A Comparative Analysis", ResearchGate, January 2017.

[4] Jin Liu, Min Li, Jianxin Wang, Fangxiang Wu, Tianming Liu, and Yi Pan, "A Survey of MRI-Based Brain Tumor Segmentation Methods", TSINGHUASCIENCEANDTECHNOLOGY, ISSN111007-02141104/101lpp578-595 Volume 19, Number 6, December 2014.

[5] Hapsari Peni Agustin Tjahyaningtijas, "Brain Tumor Image Segmentation in MRI Image", IOP Conference Series: Materials Science and Engineering, Volume 336, The 2nd International Conference on Vocational Education and Electrical Engineering (ICVEE), 9 November 2017.

[6] Amrutha Ravi, Sreejith S, "A Review on Brain Tumour Detection Using Image Segmentation", International Journal of Emerging Technology and Advanced Engineering, ISSN 2250-2459, ISO 9001:2008Certified Journal,Volume 5, Issue 6, June 2015.

[7] Jin Liu, Min Li, Jianxin Wang, Fangxiang Wu, Tianming Liu, and Yi Pan, "A Survey of MRI-Based Brain Tumor Segmentation Methods", TSINGHUA SCIENCE AND TECHNOLOGY, ISSN111007-02141104/101lpp578-595 Volume 19, Number 6, December 2014.

[8] Rash Dubey, "Region growing for MRI brain tumor volume analysis", DOI: 10.17485/ijst/2009/v2i9/2951729, Indian Journal of Science and Technology 2(9), September 2009

[9] Sheetal Mahadik1, Yashraj Naik, Darshan Onekar, Saurabh Mehta, "Systematic Toll Deduction Using Automatic Number Plate Recognition", IOSR Journal of Electronics and Communication Engineering (IOSR-JECE), e-ISSN: 2278-2834, p- ISSN: 2278 8735.Volume 11, Issue 4, Ver. II (Jul.-Aug .2016), PP 01-09 www.iosrjournals.org.

[10] Basura Fernando, Alain Trémeau, Sezer Karaoglu, "Extreme Value Theory Based Text Binarization In Documents and Natural Scenes", 2010 The 3rd International Conference on Machine Vision (ICMV 2010).

[11] Patel Janakkumar Baldevbhai, R.S. Anand, "Application of Segmentation Techniques on Atherosclerosis Images", IOSR Journal of VLSI and Signal Processing (IOSR-JVSP) e-ISSN: 2319 - 4200, p-ISSN No.: 2319 - 4197 Volume 1, Issue 6 (Mar. - Apr. 2013), PP 54-67 www.iosrjournals.org.

[12] M. Shasidhar, Sudheer raja Venishetty, B. Vijay Kumar, "MRI Brain Image Segmentation Using Modified Fuzzy C-Means Clustering Algorithm", DOI: 10.1109/CSNT.2011.102, IEEE Xplore, Communication Systems and Network Technologies (CSNT), 2011.

[13] Hau-Lee Tong, Mohammad Faizal Ahmad Fauzi and Ryoichi Komiya, "Segmentation of CT Brain Images using unsupervised clustering's", DOI: 10.1007/BF03181955, Journal of Visualization 12(2):131-138, June 2009. 